[Article]

\title{
三维介孔钴酸锌立方体的制备及其优异的储锂性能
}

\author{
甄 绪 ${ }^{2}$ 郭雪静 ${ }^{1, *}$ \\ ('南开大学环境科学与工程学院, 天津 300071; ${ }^{2} 61413$ 部队, 湖北襄阳 441003)
}

\begin{abstract}
摘要: 采用简易、温和、实际耐用的水热方法制备了新型三维介孔立方体结构的钴酸锌纳米材料。每个钴酸 锌立方体的边长大约在 3-4 $\mu \mathrm{m}$ 之间, 并由大量的纳米粒子和密集的孔隙所构成。通过氮吸附/脱附手段测试 发现所制备的钴酸锌纳米材料具有较大的比表面积 $\left(41.4 \mathrm{~m}^{2} \cdot \mathrm{g}^{-1}\right)$ 和介孔 $(6.32 \mathrm{~nm})$ 特性。使用钴酸锌纳米材料 作为锂离子电池负极, 金属锂作为正极组装锂电池并测试了材料的储锂性能。研究发现该电极材料在较高的 电流密度下循环 100 周后, 仍能呈现较高的可逆容量和超强的循环稳定性。这种优异的储锂性能主要归因于 钴酸锌纳米材料的新型结构, 这种介孔立方体结构能够加速锂离子的扩散, 增加电极与电解液的接触面积并 缓解锂离子嵌入/嵌出期间产生的体积膨胀。
\end{abstract}

关键词: 钴酸锌纳米材料; 介孔结构; 三维立方体; 锂离子电池; 负极材料

中图分类号: 0646

\section{Synthesis and Lithium Storage Performance of Three-Dimensional Mesostructured $\mathrm{ZnCO}_{2} \mathrm{O}_{4}$ Cubes}

\author{
ZHEN Xu $\quad$ GUO Xue-Jing ${ }^{1, *}$ \\ ( ${ }^{1}$ College of Environmental Science and Engineering, Nankai University, Tianjin 300071, P. R. China; \\ ${ }^{2} 61413$ Troops, Xiangyang 441003, Hubei Provnce, P. R. China)
}

\begin{abstract}
Novel three-dimensional (3D) mesostructured $\mathrm{ZnCo}_{2} \mathrm{O}_{4}$ cubes are prepared through a convenient and practical hydrothermal route combined with an annealing treatment. The as-prepared $\mathrm{ZnCo}_{2} \mathrm{O}_{4}$ cubes range from 3-4 $\mu \mathrm{m}$ in size, and are composed of a large number of nanoparticles and pores. According to $\mathrm{N}_{2}$ adsorption-desorption measurements, the as-synthesized $\mathrm{ZnCo}_{2} \mathrm{O}_{4}$ cubes have a high BET surface area (41.4 $\mathrm{m}^{2} \cdot \mathrm{g}^{-1}$ ) and mesoporous $(6.32 \mathrm{~nm})$ nature. Lithium ion batteries (LIBs) are assembled using the as-prepared $\mathrm{ZnCo}_{2} \mathrm{O}_{4}$ nanomaterial and metallic lithium as the anode and the cathode, respectively, and their lithium storage performance is investigated. The electrode material exhibits highly reversible lithium storage capacity and strong cycling stability at high current density for 100 cycles. More importantly, the $\mathrm{ZnCo}_{2} \mathrm{O}_{4}$ cube electrode still presents a relatively high specific capacity at high rate. The excellent lithium storage performance is attributed to the novel structure of the 3D mesostructured cubes, which can facilitate $\mathrm{Li}^{+}$diffusion, increase electrode/electrolyte contact area, and endure volume changes the during $\mathrm{Li}^{+}$insertion/extraction process.
\end{abstract}

Key Words: $\mathrm{ZnCo}_{2} \mathrm{O}_{4}$ nanomaterial; Mesostructure; Three-dimensional cube; Lithium ion battery; Anode material

1 引言

过渡金属氧化物由于其具有较高的理论容量
受到了广泛的关注, 使其成为锂离子电池负极材 料的有力候选 ${ }^{1,2}$ 。在过渡金属氧化物中，根据储锂

Received: October 10, 2017; Revised: December 22, 2016; Published online: December 22, 2016.

${ }^{*}$ Corresponding author. Email: 1120130162@mail.nankai.edu.cn.

The project was supported by the National Natural Science Foundation of China (12HZGJHZ01100).

国家自然科学基金(12HZGJHZ01100)资助项目

(C) Editorial office of Acta Physico-Chimica Sinica 
性质的不同，主要分为三种类型的氧化物：第一 种类型的氧化物(例如铜, 钴, 铁, 镍基氧化物)作 为锂离子电池负极材料时, 在锂离子嵌入/嵌出期 间, 通过还原反应形成氧化锂和纳米晶体状的金 属纳米颗粒 ${ }^{3}$; 第二种类型的氧化物(例如钛和锰基 氧化物) 属于嵌入型, 作为锂离子电池负极材料 时, 能够容纳一定量的锂离子, 相变成新的含锂 化合物, 并在之后的充电过程中脱出外来锂离 子, 并恢复到初始结构; 第三种类型氧化物 (如 锡, 铟和锌基氧化物) 作为锂离子电池负极材料 时, 在锂离子嵌入/嵌出期间, 会与金属锂发生反 应形成合金相, 进而提供额外的放电容量 ${ }^{4-6}$ 。而 $\mathrm{AB}_{2} \mathrm{O}_{4}$ 尖晶石型(类尖晶石型)结构的氧化物同时包 含第一种和第三种两种类型氧化物, 在能源存储 领域受到越来越多的关注 ${ }^{7}$ 。在 $\mathrm{AB}_{2} \mathrm{O}_{4}$ 尖晶石型(类 尖晶石型)结构的氧化物中, 钴酸锌由于其具有低 毒性, 低耗费性, 高可逆容量以及较强的循环稳 定性的优点, 吸引了广泛的关注 ${ }^{8,9}$ 。例如: Lou 课 题组 ${ }^{10}$ 使用单口喷嘴静电纺丝的方法制备出了多孔 钴酸锌纳米棒具有优异的电化学性能(在 $2 \mathrm{~A} \cdot \mathrm{g}^{-1}$ 电 流密度下循环 30 周, 容量能够保持在 $790 \mathrm{mAh}$. $\mathrm{g}^{-1}$ )。然而, 在循环过程中较差的电子传导性, 电 极的粉化以及较大的体积变化, 使其具有较短的 循环寿命, 阻碍了钴酸锌作为锂离子负极材料的 发展与应用。

近年来, 为了解决上述问题, 研究者们做了 诸多努力。第一种方法是构筑具有新型纳米结构 (例如层状结构, 中空结构或是介孔结构)的电极材 料 ${ }^{11}$ 。例如 Giri 课题组 ${ }^{11}$ 通过高温羟烧的方法制备 了多孔钴酸锌纳米片, 该材料作为锂离子电池负 极材料时展现了较为优异的电化学性能; 而 Hao 等 ${ }^{12}$ 使用溶剂热方法合成的介孔钴酸锌材料也具有 良好的储锂性能。这主要是由于介孔结构具有较 高容量, 较大的比表面积以及较低的密度使其在 锂离子电池中应用较为广泛。重要的是, 介孔结 构能够有效缓解在锂离子的嵌入/嵌出期间产生的 体积膨胀; 能够增强面积-体积的比值, 能够缩小 锂离子的扩散长度 ${ }^{13,14}$ 。第二种方法是制备三维电 极材料。Guo 等 ${ }^{13}$ 通过简易的水热方法结合高温炦 烧合成了介孔钴酸锌微米球, 该材料作为锂离子 电池电极材料时具有较高的比容量。在纳米结构 中, 三维立方体结构拥有稳定的结构框架, 较多 的反应活性电位和大量的电子传输通道, 能够成
为具有良好发展前景的电极材料 ${ }^{3}$ 。三维立方体纳 米结构由于其在活性材料内较短的扩散长度和增 加的电极/电解液接触面积, 使其能够加速锂离子 的扩散 ${ }^{15}$ 。第三种方法是添加一些导电材料, 提高 电极材料的导电率。例如 Gao 课题组 ${ }^{8}$ 通过添加石 墨烯制备出了超薄钴酸锌纳米片/石墨烯复合材 料。由于添加了石墨烯, 其导电性增强, 使其作 为锂离子电池负极材料时展现出了良好的循环特 性。值得注意的是三维介孔立方体结构同时拥有 介孔结构和三维立方体两种结构共同的优点, 不 需要添加任何导电性材料就能够有效地减轻纳米 材料的团聚, 缓解体积膨胀, 并提供令人满意的 电化学特性 ${ }^{16,17}$ 。因此, 发展三维介孔立方体新型 结构的纳米材料对于提升锂离子电池的性能是至 关重要的。

在本文中, 报道了一种合理的设计方案, 通 过方便、简易的水热方法及之后的高温裧烧方法 成功合成出了三维介孔立方体结构的钴酸锌纳米 材料。本文所合成的三维介孔立方体具有较大的 比表面积 $\left(41.4 \mathrm{~m}^{2} \cdot \mathrm{g}^{-1}\right)$ 以及密集的介孔孔隙 $(6.32$ $\mathrm{nm})$ 。该钴酸锌纳米材料作为锂离子电池负极材料 时具有较高的可逆循环容量和杰出的倍率性能, 其在大充放电流密度下 $\left(2000 \mathrm{~mA} \cdot \mathrm{g}^{-1}\right)$, 其容量 $\left(428 \mathrm{mAh} \cdot \mathrm{g}^{-1}\right)$ 都要高于石墨的理论容量。而该纳 米材料展现出的优异电化学性能主要归功于这种 新型的三维介孔立方体结构, 该结构能够加速锂 离子的扩散和电子的传输。并且, 这种有效地合 成策略能够拓展制备多元氧化物的合成空间。

\section{2 实验部分}

\section{1 主要试剂与仪器}

乙酸锌 $\left(\mathrm{C}_{4} \mathrm{H}_{6} \mathrm{O}_{4} \mathrm{Zn} \cdot 2 \mathrm{H}_{2} \mathrm{O}\right.$, Aladdin公司, $\left.99.9 \%\right)$, 乙酸钴 $\left(\mathrm{C}_{4} \mathrm{H}_{6} \mathrm{CoO}_{4} \cdot 4 \mathrm{H}_{2} \mathrm{O}\right.$, Aladdin 公司, $\left.99.9 \%\right)$, 碳酸氢钠 $\left(\mathrm{NaHCO}_{3}\right.$, Aladdin 公司, $\left.99.9 \%\right)$, 尿素 $\left(\mathrm{C}_{2} \mathrm{O}_{2} \mathrm{~N}_{4} \mathrm{H}_{4}, \quad\right.$ Aladdin 公司, $\left.99.9 \%\right)$, 无水乙醇 $\left(\mathrm{C}_{2} \mathrm{H}_{6} \mathrm{O}, \mathrm{AR}\right.$ (沪试), 99.7\%)。以上试剂均为分析纯。

\section{2 钴酸锌纳米材料的制备}

将 $0.66 \mathrm{~g}$ 乙酸锌、 $1.5 \mathrm{~g}$ 乙酸钴, $0.45 \mathrm{~g}$ 碳酸氢 钠, $0.63 \mathrm{~g}$ 尿素溶于 $15 \mathrm{~mL}$ 乙醇和 $15 \mathrm{~mL}$ 蒸馏水 中, 得到混合溶液。将混合溶液放入 $50 \mathrm{~mL}$ 聚四氟 乙烯反应釜中磁力搅拌 $30 \mathrm{~min}$, 之后将反应釜放入 $180^{\circ} \mathrm{C}$ 烘箱中, 放置 $24 \mathrm{~h}$ 之后取出, 待室温冷却后 将底部沉淀取出, 用乙醇和蒸馏水分别洗涤 3 次并 
收集沉淀, 再在 $120{ }^{\circ} \mathrm{C}$ 下干燥 $8 \mathrm{~h}$, 得到 $\mathrm{ZnCo}_{2} \mathrm{O}_{4}$ 前驱体。将前驱体置于管式炉, 并在空气中以 $2{ }^{\circ} \mathrm{C} \cdot \mathrm{min}^{-1}$ 的速率升温至 $600{ }^{\circ} \mathrm{C}$, 恒温炦烧 $2 \mathrm{~h}$, 制 得黑色 $\mathrm{ZnCo}_{2} \mathrm{O}_{4}$ 粉末。

\section{3 材料分析}

所获得的样品使用 $\mathrm{X}$ 射线粉末衍射分析 (XRD, 所使用的测试设备为 Rigaku D/Max III, 德 国生产，测试所采用 $\mathrm{Cu}$ 的 $K_{\alpha}$ 辐射(波长为 0.154 $\mathrm{nm})$, 以连续的扫描方式测试, 范围为 $2 \theta=3^{\circ}-$ $80^{\circ}$, 扫描速率为 $\left.2\left(^{\circ}\right) \cdot \mathrm{min}^{-1}\right)$; 扫描电子显微镜 (SEM, 测试样品的扫描电镜图采用的扫描电镜设 备为FEI Nanosem 430、HITACHI S-3500N 和 JEOL$7500 \mathrm{~F}$ 型, 日本生产); 透射电镜(TEM, 所使用的 透射电镜图由 FEI Tecnai G2F-20 和 JEM 3010 透射 电镜测试得到, 日本生产); 比表面与孔径分析 (BET; 比表面分析采用 Quantachrome NOVA 2000e 比表面分析仪, 英国生产。比表面数据根据BET方 程计算得出, 孔径分布由 BJH方法计算得到)。

\section{4 电池制作和测试}

工作电极是由活性物质、乙炔黑和聚四氟乙 烯构成, 三种物质组成的质量比为 $75: 15: 10$ 。平 均每个极片的重量约为 $2 \mathrm{mg}$ 。在超级净化真空手 套箱 $(S u p e r(1220 / 750 / 900))$ 米开罗那(中国)有限公 司)中组装模拟电池。在电池测试过程中, 金属锂 作为对电极和参比电极。隔膜多采用多孔的聚丙 稀隔膜 (Celgard 2400), 电解液为 $1 \mathrm{~mol} \cdot \mathrm{L}^{-1}$ 的 $\mathrm{LiPF}_{6} / \mathrm{EC}+\mathrm{EMC}+\mathrm{DMC}(1: 1: 1$ (体积分数) $)$ (JN908), 铜片作为集流体。电池组装好后封蜡, 室温静置 $12 \mathrm{~h}$ 后进行电化学性能测试。所制备的 模拟电池在 Land 2001A 电池测试系统(武汉金诺电 子有限公司制造)上进行充放电测试, 其充放电电 压范围为 $0.001-3.00 \mathrm{~V}$ 。测试均在室温下进行。电
化学阻抗(EIS)测试在本实验中采用 Zahner-Elektrik IM6e 型电化学工作站进行电化学阻抗测试。其扫 描频率范围为 $100 \mathrm{kHz}-10 \mathrm{mHz}$, 电位波幅 $5 \mathrm{mV}$ 。 测试温度为 $25^{\circ} \mathrm{C}$ 。

\section{3 结果与讨论}

\section{1 材料物相及形貌分析}

图 1 展现了所制备样品的 X射线衍射 (XRD) 图, 从图中可以清晰地观察到所合成材料的所有 衍射峰都能够很好地与尖晶石型钴酸锌的标准卡 片 (JCPDS 23-1390) 所对应, 并没有其它任何杂 峰, 这就说明所制备的材料是纯相的尖晶石型钴 酸锌材料, 并且在衍射图中可以看出所合成的材 料呈现出良好的结晶度。从钴酸锌的晶体结构中 (图 1B)可以看出, $\mathrm{Zn}^{2+}$ 占据了四面体格位, $\mathrm{Co}^{3+}$ 占 据了八面体格位。

扫描电镜图(SEM, 图 2)显示出了所制备的钴 酸锌纳米材料的形貌和微观结构。从 SEM 图 2A 中 可以看出, 所制备的钴酸锌材料是十分均匀的立 方体, 每个立方体的大小在 3-4 $\mu \mathrm{m}$ 之间。图 2B 展示了单个立方体的形态, 可以从中看出每个钴 酸锌立方体是由众多的纳米粒子构成的, 并且这 些纳米粒子之间形成了密集的孔隙。

为了进一步观察所制备样品的形貌和结构, 本文测试了所制备钴酸锌纳米材料的透射电镜图 (TEM, 图 3)。从图 3A 中可以明显的观察到钴酸锌 纳米材料呈立方体状, 这也与 SEM 图是相符合 的。而在图 3B 中立方体的边缘显现出了较为模糊 的纳米粒子, 而图 $3 \mathrm{C}$ 则是清晰的展现了立方体的 边缘, 这也就进一步证明了钴酸锌立方体是由众 多纳米粒子组成的, 并且这些纳米粒子之间具有 密集的孔隙。在超高倍数下的 TEM 图(图 3D)中,
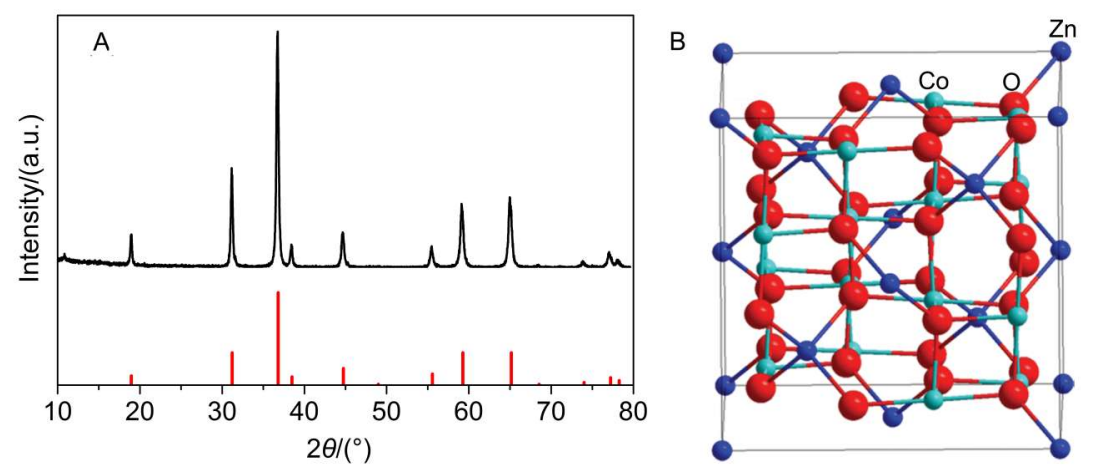

图 1 所制备钴酸锌纳米材料的 $\mathbf{X}$ 射线衍射图谱(A)和晶体结构图(B)

Fig.1 XRD patterns (A) and crystal structure (B) of as-prepared $\mathrm{ZnCo}_{2} \mathrm{O}_{4}$ nanomaterials 

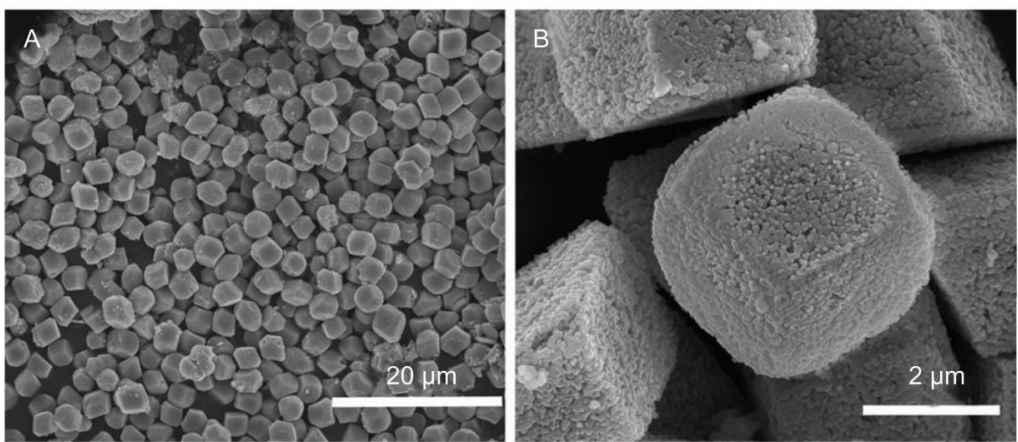

图 2 所制备钴酸锌纳米材料的低倍(A)和高倍(B)扫描电镜图

Fig.2 Low magnification (A) and high magnification (B) scanning electron microscopy images (SEM) of as-prepared $\mathrm{ZnCo}_{2} \mathrm{O}_{4}$ nanomaterials
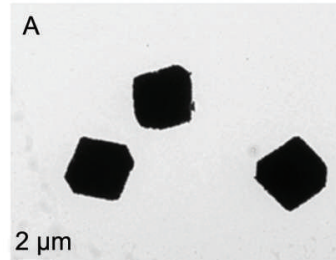

B

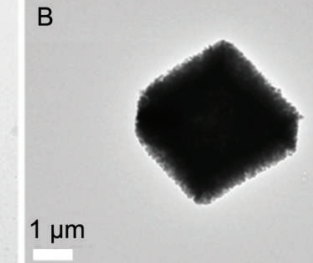

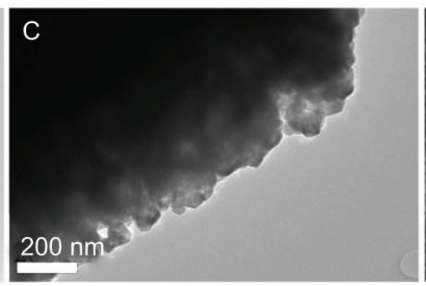

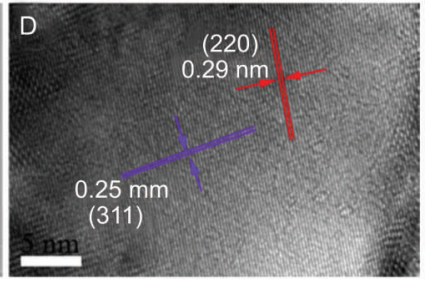

图 3 所制备钴酸锌纳米材料的低倍 $(\mathrm{A}-\mathrm{C})$ 和高倍(D)透射电镜图

Fig.3 Low-resolution transmission electron microscopy (TEM) (A-C) and high-resolution TEM (D) images of the as-prepared $\mathrm{ZnCo}_{2} \mathrm{O}_{4}$ materials

可以观察到 0.25 和 $0.29 \mathrm{~nm}$ 距离的晶格, 其相对应 的是尖晶石型钴酸锌的(311)面和(220)面, 这与 XRD 的结果也是相符合的。

为了进一步确定所制备的钴酸锌立方体的比 表面积大小以及多孔结构, 本文使用了氮吸附-脱 附手段进行测试。从图 4A 中可以看出, 所制备的 钴酸锌纳米材料的等温线呈现出典型的 IV 型, 这 就证明了该材料具有介孔结构 ${ }^{18,19}$ 。而孔径分布曲 线(图 4B)表明了所制备的材料的孔径大约在 5-7 $\mathrm{nm}$, 又进一步证明了所制备的钴酸锌纳米材料具 有介孔结构。根据测试结果该材料的比表面积,

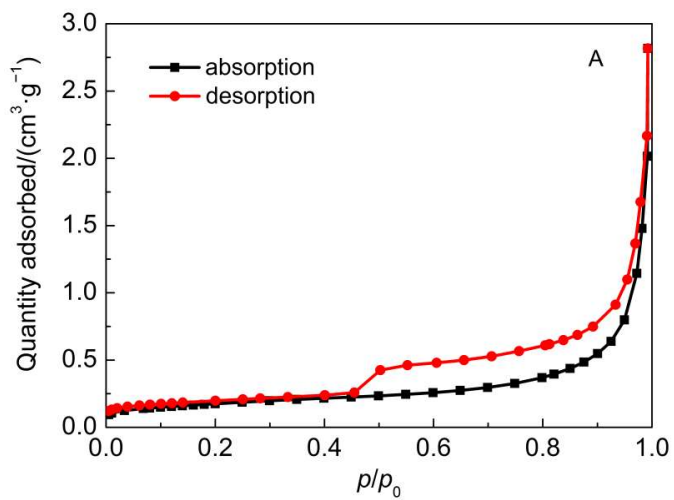

孔体积和平均孔径分别为 $41.4 \mathrm{~m}^{2} \cdot \mathrm{g}^{-1}, 0.135 \mathrm{~cm}^{3}$. $\mathrm{g}^{-1}$ 和 $6.32 \mathrm{~nm}$ 。值得注意的是, 具有较大的比表面 积的介孔结构能够提供较高的电极/电解液的接触 面积 ${ }^{20,21}$, 能够增加锂离子嵌入/嵌出反应的活性位 点, 进而在持续充放电期间提供高循环容量 ${ }^{22,23}$ 。

\section{2 材料电化学性能分析}

为了进一步分析复合材料的电化学性能, 本 文将所制备的样品应用在了锂离子电池的负极材 料中。图 5 展现了钴酸锌电极在 $0.001-3 \mathrm{~V}, 0.5$ $\mathrm{mV} \cdot \mathrm{s}^{-1}$ 的扫速下, 从第一圈到第六圈的循环伏安 $(\mathrm{CV})$ 曲线。在第一循环中, 钴酸锌电极在 $~ 0.6 \mathrm{~V}$ 的

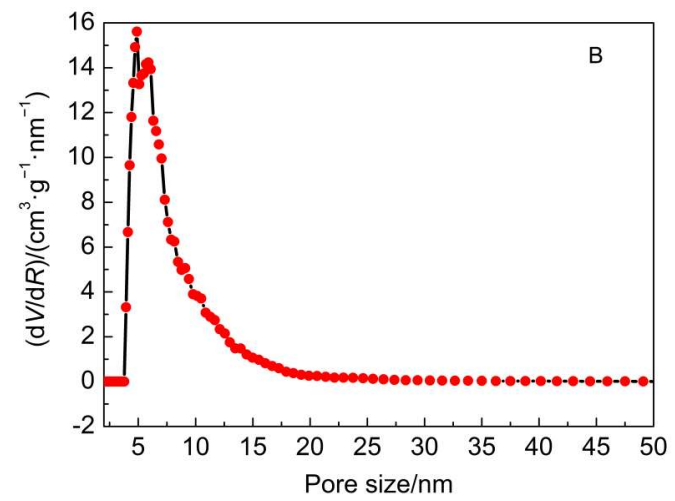

图 4 所制备的钴酸锌纳米材料的氮吸附/脱附曲线(A)和孔径分布曲线(B)

Fig.4 $\mathrm{N}_{2}$ absorption-desorption isotherm (A) of the as-prepared $\mathrm{ZnCo}_{2} \mathrm{O}_{4}$ microspheres and the pore distribution (B) 


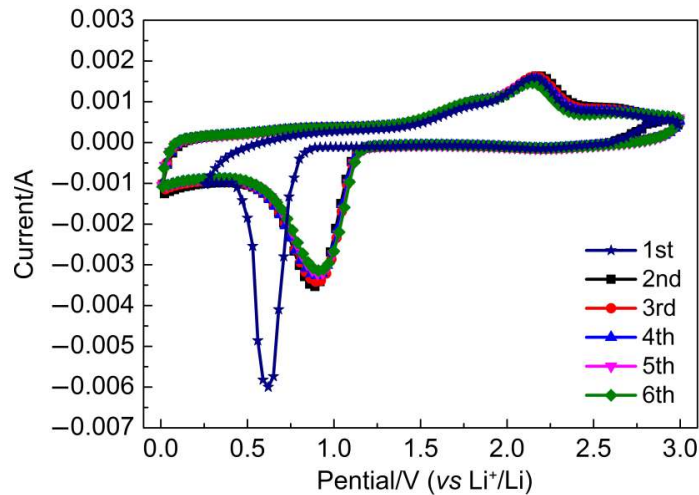

图 5 在 $0.001-3.0 \mathrm{~V}$ 电压下 $0.5 \mathrm{mV} \cdot \mathrm{s}^{-1}$ 扫描速率时的 循环伏安曲线

Fig.5 Cyclic voltammetry (CV) curves at a scan rate of $0.5 \mathrm{mV} \cdot \mathrm{s}^{-1}$ in the voltage window of $0.001-3.0 \mathrm{~V}$

位置出现了明显的、不可逆的还原峰, 这主要是 因为钴酸锌被还原成金属 $\mathrm{Co}$ 与 $\mathrm{Zn}$, 其中还伴随着 一些与有机电解质分解为固体电解质界面(SEI 膜) 相关的不可逆反应; 在 $~ 1.7$ 和 $2.1 \mathrm{~V}$ 处出现了两个 氧化峰, 分别对应于 $\mathrm{Zn}$ 氧化成 $\mathrm{Zn}^{2+}$ 和 $\mathrm{Co}$ 氧化成 $\mathrm{Co}^{3+}$, 并伴随 $\mathrm{Li}^{+}$脱出的过程 ${ }^{24}$ 。与第一次循环相 比, 第二到第六次循环之后的 $\mathrm{CV}$ 曲线中的还原峰 转移到了 $1.1 \mathrm{~V}$ 位置, 且强度减弱, 这主要是因为 之后的循环中较少形成 SEI 膜。第二到第六次的 $\mathrm{CV}$ 循环曲线基本重叠, 这说明了 $\mathrm{Li}^{+}$在电极材料中 的嵌脱电化学反应可逆性良好, 且电极材料的结 构十分稳定。根据上述分析, 钴酸锌做锂离子电 池负极材料时的充放电反应分别为 ${ }^{25,26}$ :

放电反应:

$$
\begin{aligned}
& \mathrm{ZnCo}_{2} \mathrm{O}_{4}+8 \mathrm{Li}^{+}+8 \mathrm{e}^{-} \rightarrow \mathrm{Zn}+2 \mathrm{Co}+4 \mathrm{Li}_{2} \mathrm{O} \\
& \mathrm{Zn}+\mathrm{Li}^{+}+\mathrm{e}^{-} \rightarrow \mathrm{LiZn} \\
& \mathrm{ZnO}+2 \mathrm{Li}^{+}+2 \mathrm{e}^{-} \rightarrow \mathrm{Zn}+\mathrm{Li}_{2} \mathrm{O} \\
& \mathrm{CoO}+2 \mathrm{Li}^{+}+2 \mathrm{e}^{-} \rightarrow \mathrm{Co}+\mathrm{Li}_{2} \mathrm{O} \\
& \mathrm{Co}_{3} \mathrm{O}_{4}+2 \mathrm{Li}^{+}+2 \mathrm{e}^{-} \rightarrow 3 \mathrm{CoO}+\mathrm{Li}_{2} \mathrm{O} \\
& \text { 充电反应: } \\
& \mathrm{LiZn} \rightarrow \mathrm{Zn}+\mathrm{Li}^{+}+\mathrm{e}^{-} \\
& \mathrm{Zn}+\mathrm{Li}_{2} \mathrm{O} \rightarrow \mathrm{ZnO}+2 \mathrm{Li}^{+}+2 \mathrm{e}^{-} \\
& \mathrm{Co}+\mathrm{Li}_{2} \mathrm{O} \rightarrow \mathrm{CoO}+2 \mathrm{Li}^{+}+2 \mathrm{e}^{-} \\
& 3 \mathrm{CoO}+\mathrm{Li}_{2} \mathrm{O} \rightarrow \mathrm{Co}_{3} \mathrm{O}_{4}+2 \mathrm{Li}^{+}+2 \mathrm{e}^{-}
\end{aligned}
$$

图 6A 显示了钴酸锌电极在 $0.001-3 \mathrm{~V}$ 电压 范围, $500 \mathrm{~mA} \cdot \mathrm{g}^{-1}$ 电流密度下, 循环第 1 周, 第 2 周，第 10 周，第 100 周的充放电曲线。该电极在 初始周呈现出较高的充放电容量(676/1041 mAh. $\mathrm{g}^{-1}$ ), 相对应的不可逆容量损失大约为 $35 \%$, 这 主要是固体电解质界面 (SEI)膜和金属的锌、钴

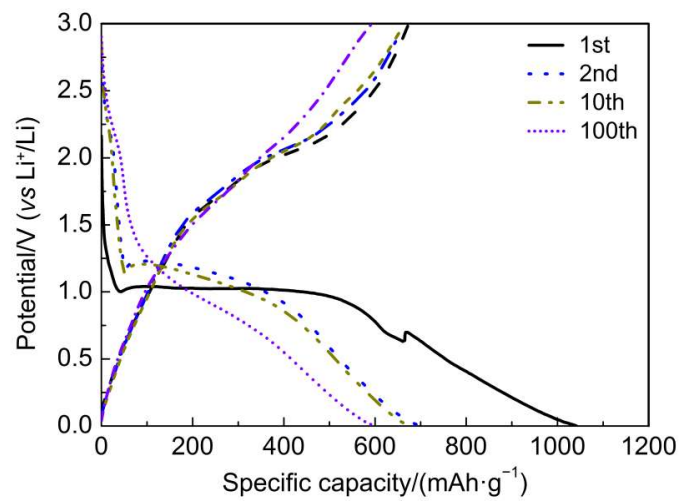

图 6 所制备的钴酸锌纳米材料电极在 $500 \mathrm{~mA} \cdot \mathrm{g}^{-1}$ 电流密度下的充放电曲线

Fig.6 Specific discharge/charge capacities of as-prepared $\mathrm{ZnCo}_{2} \mathrm{O}_{4}$ nanomaterials at the current density of $500 \mathrm{~mA} \cdot \mathrm{g}^{-1}$

到初始氧化物的不完全的恢复。在第一次循环之 后放电平台 $(\sim 1 \mathrm{~V})$ 的消失表明了稳定的 SEI 膜在第 一周循环之后形成。从第二周循环之后，较长的 充放电平台有一个较大的倾斜在 1.35 和 $0.72 \mathrm{~V}$ 之 间。第一周循环之后(第 2 周，第 10 周，第 100 周) 产生的这种充放电曲线的较好的重叠也证明了在 锂离子嵌入/嵌出期间，材料具有优异的电化学可 逆性和稳定性。

更重要的是, 钴酸锌纳米材料电极在 $500 \mathrm{~mA}$. $\mathrm{g}^{-1}$ 充放电流密度下, 循环 100 周之后，仍能保持较 高的容量 $600 \mathrm{mAh} \cdot \mathrm{g}^{-1}$, 这也就展现出了电极材料 的高可逆容量以及杰出的循环特性(图 7A)。图 7B 展示了钴酸锌纳米材料在不同的电流倍率下的充 放电倍率性能。分别在 500、1000、2000、3000、 $5000 \mathrm{~mA} \cdot \mathrm{g}^{-1}$ 的电流密度下, 该纳米材料的可逆放 电容量分别为 $775 、 627 、 428 、 253$ 和 $120 \mathrm{mAh}$. $\mathrm{g}^{-1}$ 。值得注意的是, 该电极在 $2000 \mathrm{~mA} \cdot \mathrm{g}^{-1}$ 的电流 倍率下的容量都要高于商业石墨的理论容量(372 $\left.\mathrm{mAh} \cdot \mathrm{g}^{-1}\right)$ 。更重要的是, 当电流密度突然转回 $1000 \mathrm{~mA} \cdot \mathrm{g}^{-1}$ 时, 原始的容量仍然能够保持, 这就 证明了钴酸锌纳米材料具有超强的稳定性和可逆 性 25,27 。通过比较已报道的钴酸锌纳米材料的电化 学性能 ${ }^{28-30}$, 发现本文中的三维介孔钴酸锌立方体 在大电流密度下, 循环 100 周之后保持的比容量仍 然高于文献中报道的不同形貌钴酸锌纳米材料在 小电流密度下的循环容量。

为了进一步确定三维立方体钴酸锌电极材料 的形貌和结构稳定性, 本文测试了钴酸锌电极材 料在充放电 100 圈之后的扫描电镜图(图 8)。从图 $8 \mathrm{~A}$ 中可以观察到, 基本的三维立方体形貌仍然存 

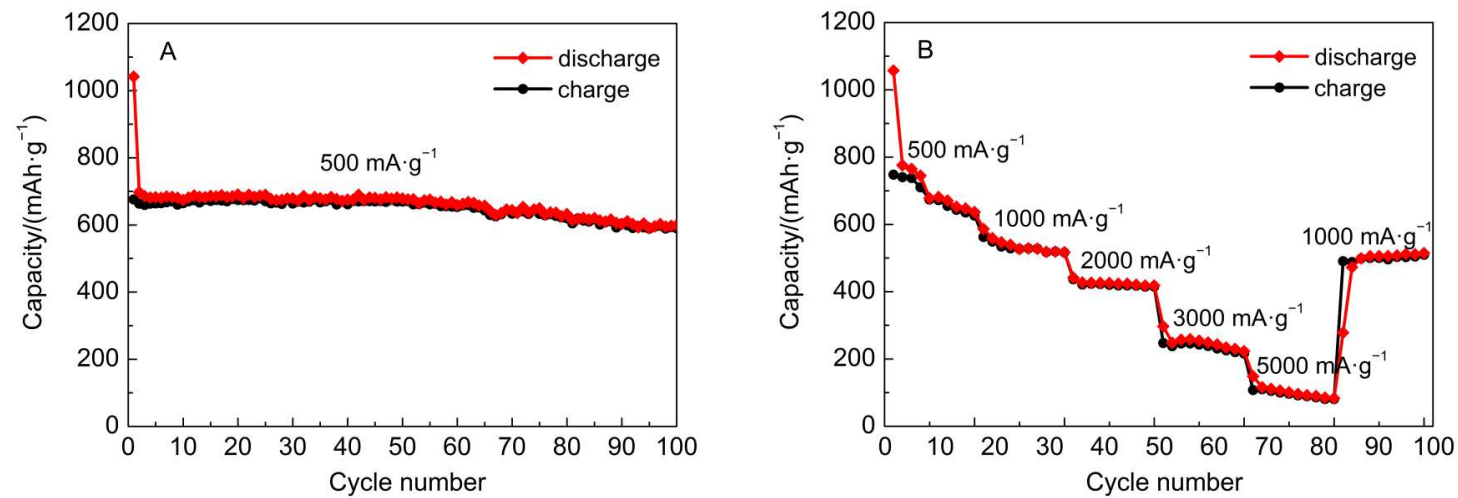

图 7 所制备的钴酸锌纳米材料电极在 $500 \mathrm{~mA} \cdot \mathrm{g}^{-1}$ 电流密度下的循环性能(A); 电极在不同电流密度下的充放电比容量(B)

Fig.7 Cyclic performance (A) of as-prepared $\mathrm{ZnCo}_{2} \mathrm{O}_{4}$ nanomaterials at the current density of $500 \mathrm{~mA} \cdot \mathrm{g}^{-1}$; rate performances $(B)$ at different current densities

表 1 已报道不同形貌的钴酸锌纳米材料的电化学性能的比较

Table 1 Compared electrochemical performance of reported $\mathrm{ZnCo}_{2} \mathrm{O}_{4}$ nanostructures with different morphologies

\begin{tabular}{ccccc}
\hline Sample & Current density $/\left(\mathrm{mA} \cdot \mathrm{g}^{-1}\right)$ & Cycles & Capacity $/\left(\mathrm{mAh} \cdot \mathrm{g}^{-1}\right)$ & Reference \\
\hline 3D mesostructure $\mathrm{ZnCo}_{2} \mathrm{O}_{4}$ cubes & 500 & 100 & 600 & this work \\
$\mathrm{ZnCo}_{2} \mathrm{O}_{4}$ polypyrrole & 100 & 100 & 600 & 28 \\
$\mathrm{ZnCo}_{2} \mathrm{O}_{4}$ nanoparticles & 100 & 70 & 300 & 29 \\
mesostructured $\mathrm{ZnCo}_{2} \mathrm{O}_{4}$ & 100 & 100 & 300 & 30 \\
\hline
\end{tabular}

在, 而从图 8B 中可以清晰地看出钴酸锌的三维立 方体结构，这也就证明了三维介孔立方体结构能 够缓解锂离子嵌入/嵌出引起的体积膨胀, 能够保
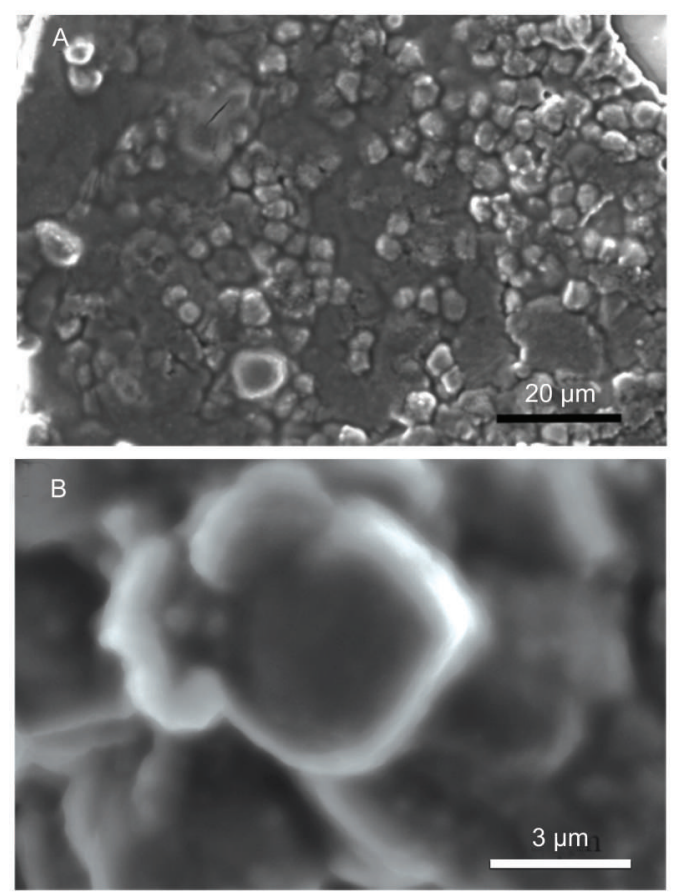

图 8 所制备的钴酸锌电极材料循环 100 圈之后的 扫描电镜图

Fig.8 SEM images of as-prepared $\mathrm{ZnCo}_{2} \mathrm{O}_{4}$ electrode materials after 100 cycles
持结构的稳定性, 进而保持较长的循环寿命。

为了进一步分析钴酸锌纳米材料的电化学性 能，本文测试了钴酸锌纳米材料做工作电极时的 交流阻抗并模拟了相对应的电路图(图 9)。样品在 高频区显示出一个半圆，在低频区显示出一条斜 线。其中高频区的半圆对应电极/电解液表面的电 荷转移阻抗 ${ }^{9}$, 低频区的直线对应的电化学过程中

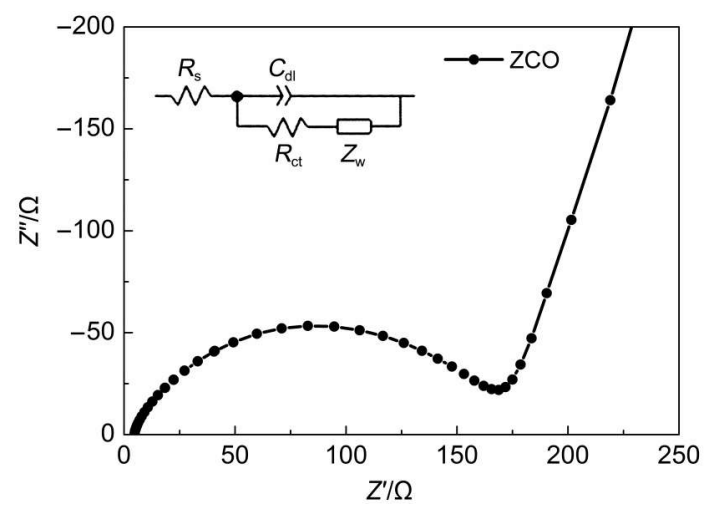

图9所制备的钴酸锌纳米材料电极的交流阻抗图以及 相对应的模拟电路图

Fig.9 Nqyuist plots for the EIS of as-prepared $\mathrm{ZnCo}_{2} \mathrm{O}_{4}$ nanomaterials and the corresponding equivalent circuit mode

$\mathrm{ZCO}$ represents $\mathrm{ZnCo}_{2} \mathrm{O}_{4} ; R_{\mathrm{s}}$ is the total resistance of the electrolyte; $R_{\mathrm{ct}}$ is the charge-transfer resistance; $C_{\mathrm{dl}}$ represents the double-layer resistance; and $\mathrm{Z}_{\mathrm{w}}$ is the Warburg impedance. 


\begin{tabular}{|c|c|c|c|}
\hline \multicolumn{4}{|c|}{$\begin{array}{l}\text { Table } 2 \text { Physicochemical properties measured and } \\
\text { calculated from EIS spectra (in Fig.9) }\end{array}$} \\
\hline Sample & $R_{\mathrm{s}} / \Omega$ & $R_{\mathrm{c}} / \Omega$ & Reference \\
\hline $3 \mathrm{D}$ mesostructure $\mathrm{ZnCo}_{2} \mathrm{O}_{4}$ cubes & 3.82 & 157.03 & this work \\
\hline $\mathrm{ZnCo}_{2} \mathrm{O}_{4}$ nanorods & 5.14 & 192.00 & 33 \\
\hline orderedmesostructure $\mathrm{ZnCo}_{2} \mathrm{O}_{4}$ & 3.98 & 190.60 & 23 \\
\hline $\mathrm{ZnCo}_{2} \mathrm{O}_{4}$ polyhedron & 12.03 & 1284.73 & 34 \\
\hline
\end{tabular}

$\mathrm{Li}^{+}$的扩散速率 ${ }^{31,32}$ 。钴酸锌电极的 EIS 图在高频区 呈现出一个较小的半圆, 在低频区显示出一条具 有较大斜率的直线。在模拟的电路图中, $R_{\mathrm{s}}$ 和 $R_{\mathrm{ct}}$ 分别代表电解质阻抗和电荷转移阻抗, 相应的值 在表 1 中列出。通过与已报道的文章对比 ${ }^{23,33,34}$, 三 维立方体钴酸锌的 $R_{\mathrm{s}}$ 和 $R_{\mathrm{ct}}$ 值最小, 这表明了本文 所制备的纳米材料作电极时具有较小的阻抗值和 较大的锂离子扩散率 ${ }^{27,35}$, 这也就进一步证明了介 孔立方体结构能够有效地提高电子的传输和锂离 子的扩散, 这就使得介孔钴酸锌立方体具有较高 的充放电容量 ${ }^{36}$ 。

\section{4 结 论}

本文使用简易、温和的方法构筑了介孔钴酸 锌立方体纳米材料。所制备的钴酸锌纳米材料作 为锂离子电池负极时, 展现了较高的可逆容量, 优异的循环性能以及高倍率性能。这种杰出的电 化学性能主要归因于钴酸锌纳米材料的新型结 构, 这种介孔立方体结构能够加速锂离子的扩 散, 增加电极与电解液的接触面积, 缓解锂离子 嵌入/嵌出期间产生的体积膨胀。这种温和的合成 策略能够适用于具有较高锂离子存储性能的过渡 金属纳米材料的制造和优化。

\section{References}

(1) Tang, Y. P.; Yuan, S.; Guo, Y. Z.; Huang, R. A.; Wang, J. H.; Yang, B.; Dai, Y. N. Acta Phys. -Chim. Sin. 2016, 32, 2280. [唐 艳平, 元 莎, 郭玉忠, 黄瑞安, 王剑华, 杨 斌, 戴永年. 物理 化学学报, 2016, 32, 2280.] doi: 10.3866/PKU. WHXB201605124

(2) Tang, Z. Y.; Feng, J. J. Acta Phys. -Chim. Sin. 2003, 19, 1025. [唐致远, 冯季军. 物理化学学报, 2003, 19, 1025.] doi: 10.3866/PKU.WHXB20031108

(3) Gao, G. X.; Wu, H. B.; Ding, S. J.; Lou, X. W. Small 2015, 11, 432. doi: $10.1002 / \mathrm{sm} 11.201400152$

(4) Bai, J.; Li, X.; Liu, G.; Qian, Y.; Xiong, S. Adv. Funct. Mater. 2014, 24, 3012. doi: 10.1002/adfm.201303442
(5) Wang, M.; Cheng, L. M.; Li, Q. B.; Chen, Z. W.; Wang, S. L. Phys. Chem. Chem. Phys. 2014, 16, 21742. doi: 10.1039/ c4cp03407d

(6) Woo, M. A.; Kim, T. W.; Kim, I. Y.; Hwang, S. J. Solid State Ionics 2011, 182, 91. doi: 10.1016/j.ssi.2010.10.025

(7) Bresser, D.; Paillard, E.; Kloepsch, R.; Krueger, S.; Fiedler, M.; Schmitz, R.; Baither, D.; Winter, M.; Passerini, S. Adv. Energy Mater: 2013, 3, 513. doi: 10.1002/aenm.201200735

(8) Gao, G. X.; Wu, H. B.; Dong, B. T.; Ding, S. J.; Lou, X. W. Adv. Sci. 2015, 2, 1400014. doi: 10.1002/advs. 201400014

(9) Zhou, L.; Wu, H. B.; Zhu, T.; Lou, X. W. J. Mater. Chem. 2012, 22, 827. doi: 10.1039/c1jm15054e

(10) Luo, W.; Hu, X. L.; Sun, Y. M.; Huang, Y. H. J. Mater. Chem. 2012, 22, 8916. doi: 10.1039/c2jm00094f

(11) Giri, A. K.; Pal, P.; Ananthakumar, R.; Jayachandran, M.; Mahanty, S.; Panda, A. B. Cryst. Growth Des. 2014, 14, 3352. doi: $10.1021 / \operatorname{cg} 500282 \mathrm{n}$

(12) Hao, S. J.; Zhang, B. W.; Ball, S.; Copley, M.; Xu, Z. C.; Srinivasan, M.; Zhou, K.; Mhaisalkar S.; Huang, Y. Z. J. Power Sources 2015, 294, 112. doi: 10.1016/j.jpowsour.2015.06.048

(13) Guo, L. Y.; Ru, Q.; Song, X.; Hu, S. J.; Mo, Y. D. RSC Adv. 2015, 5, 19241. doi: 10.1039/c4ra15553j

(14) Hung, T. F.; Mohamed, S. G.; Shen, C. C.; Tsai, Y. Q.; Chang, W. S.; Liu, R. S. Nanoscale 2013, 5, 12115. doi: 10.1039/ c3nr04271e

(15) Liu, X. H.; Zhang, J.; Wang, L. W.; Yang, T. L.; Guo, X. Z.; Wu, S. H.; Wang, S. R. J. Mater. Chem. 2011, 21, 349. doi: 10.1039/ c0jm01800g

(16) Hou, L.; Lian, L.; Zhang, L.; Pang, G.; Yuan, C.; Zhang, X. Adv Funct. Mater. 2015, 25, 238. doi: 10.1002/adfm.201402827

(17) Li, J.; Xiong, S.; Li, X.; Qian, Y. Nanoscale 2013, 5, 2045. doi: $10.1039 / \mathrm{c} 2 \mathrm{nr} 33576 \mathrm{j}$

(18) Zhang, C.; Huang, B.; Qian, L. H.; Yuan, S. L.; Wang, S.; Chen, R. ChemPhysChem 2016, 17, 98. doi: 10.1002/cphc.201500854

(19) Sun, L.; Zhao, Z.; Zhou, Y.; Liu, L. Nanoscale 2012, 4, 613. doi: $10.1039 / \mathrm{c} 1 \mathrm{nr} 11411 \mathrm{e}$

(20) Qu, Q. T.; Fu, L. J.; Zhan, X. Y.; Samuelis, D.; Maier, J.; Li, L.; Tian, S.; Li, Z. H., Wu, Y. P. Energy Environ. Sci. 2011, 4, 3985. doi: 10.1039/c0ee00673d

(21) Yang, X. H.; Li, Z.; Sun, C. H.; Yang, H. G.; Li, C. Z. Chem Mater. 2011, 23, 3486. doi: 10.1021/cm2008768

(22) Huang, Y. H. Acta Phys. -Chim. Sin. 2016, 32, 816. [黄云辉. 物 理化学学报, 2016, 32, 816.] doi: 10.1021/cm2008768

(23) Zhao, R. Z.; Li, Q.; Wang, C. X.; Yin, L. W. Electrochim. Acta 2016, 197, 58. doi: 10.1016/j.electacta.2016.03.047

(24) Mo, Y. D.; Ru, Q.; Chen, J. F.; Song, X.; Guo, L. Y.; Hu, S. J.; Peng, S. M. J. Mater. Chem. A 2015, 3, 19765. doi: 10.1039/ C5TA05931C

(25) Wang, Y. J.; Zhang, Y. Z.; Ou, J. K.; Zhao, Q.; Liao, M.; Xiao, D. $R S C$ Adv 2016, 6, 547. doi: 10.1039/c5ra21916g

(26) Song, X.; Ru, Q.; Zhang, B.; Hu, S.; An, B. J. Alloy. Compd. 
2014, 585, 518. doi: 10.1016/j.jallcom.2014.03.190

(27) Xu, J. M.; He, L.; Wang, Y. J.; Zhang, C. J.; Zhang, Y. H. Electrochim. Acta 2016, 191, 417. doi: 10.1016/j. electacta.2016.01.023

(28) Huang, L.; Waller, G. H.; Ding, Y.; Chen, D.; Ding, D.; Xi, P.; Wang, Z. L.; Liu, M. Nano Energy 2015, 11, 64. doi: 10.1016/j. nanoen.2014.09.027

(29) Rai, A. K.; Thi, T. V.; Paul, B. J.; Kim, J. Electrochim. Acta 2014, 146, 577. doi: 10.1016/j.electacta.2014.09.079

(30) Zhong, X. B.; Wang, H. Y.; Yang, Z. Z.; Jin, B.; Jiang, Q. C. J. Power Sources 2015, 296, 298. doi: 10.1016/j. jpowsour.2015.07.047

(31) Zhang, Z. C.; Chen, Y. F.; He, S.; Zhang, J. C.; Xu, X. B.; Yang, Y.; Nosheen, F.; Saleem, F.; He, W.; Wang, X. Angew. Chem.
Int. Edit. 2014, 53, 12517. doi: 10.1002/anie.201406484

(32) Luo, W.; Huang, L.; Guan, D. D.; He, R. H.; Li, F.; Mai, L. Q. Acta Phys. -Chim. Sin. 2016, 32, 1999. [罗 雯, 黄 否, 关豆 豆, 贺汝涵, 李 枫, 麦立强. 物理化学学报, 2016, 32, 1999.] doi: 10.3866/PKU.WHXB20160532

(33) Liu, H. W.; Wang, J. Electrochim. Acta 2013, 92, 371. doi: 10.1016/j.electacta.2014.09.079

(34) Ru, Q.; Song, X.; Mo, Y. D.; Guo, L. Y.; Hu, S. J. J. Alloy. Compd. 2016, 654, 586. doi: 10.1016/j.jallcom.2015.09.105

(35) Varzi, A.; Bresser, D.; Zamory, J.; Müller, F.; Passerini, S. $A d v$. Energy Mater. 2014, 4, 1400054. doi: 10.1002/aenm.201400054

(36) Zou, F.; Hu, X.; Li, Z.; Qie, L.; Hu, C.; Zeng, R.; Jiang, Y.; Huang, Y. Adv. Mater. 2014, 26, 6622. doi: 10.1002/ adma.201402322 